\title{
Treatment Strategy Using mtor Pathway in Incurable Prostate Cancer
}

\author{
Su Hwa Jang ${ }^{1,2}$, Jaekyoung Park ${ }^{3}$ and Jung Ki Jo ${ }^{3 *}$ \\ ${ }^{1}$ Biomedical Research Institute, Korea \\ ${ }^{2}$ Department of Biomedical Science, Graduate School of Biomedical Science and Engineering, Hanyang University, South Korea \\ ${ }^{3}$ Department of Urology, College of Medicien, Hanyang University, Korea
}

*Corresponding author: Jung Ki Jo, Department of Urology, Hanyang University Seoul Hospital, Wangsimni-ro 222, Seongdong-gu, Seoul 04763, Korea.

To Cite This Article: Su Hwa Jang, Jaekyoung Park, Jung Ki Jo, Treatment Strategy Using mtor Pathway in Incurable Prostate Cancer. Am J Biomed Sci \& Res. 2021 - 12(2). AJBSR.MS.ID.001723. DOI: 10.34297/AJBSR.2021.12.001723.

Received: 眥 February 17, 2021; Published: 制 March 01, 2021

\begin{abstract}
In spite of androgen deprivation therapy and novel hormone therapy such as enzalutamide or abiraterone has possibility of various regimens, there is still barrier to expect a complete response (CR) in castrate-resistant prostate cancer (CRPC) patients. In these cases, a new strategy using drugs targeting PI3K/AKT/mTOR pathway is necessary. In conclusion, drugs or biomarkers targeting PI3K/AKT/mTOR pathway in patients with CRPC may be used to establish a novel treatment strategy. Patients with prostate cancer are generally known to have a good prognosis; however, it is highly dependent on the risk group. Although the introduction of androgen deprivation therapy and the development of new hormone therapy drugs such as enzalutamide or abiraterone has made various treatment regimens possible, it is still difficult to expect a complete response (CR) in castrate-resistant prostate cancer (CRPC) patients. Despite of cabazitaxel development, patients who are unresponsive to docetaxel and cabazitaxel are classified as patients with incurable prostate cancer. To treat this incurable cancer, drugs with different mechanism of actions are considered. Recently, PARP inhibitors which target glutamine metabolism, a key source of carbon, are anticipated to be introduced in the clinical practice. Nevertheless, treatment strategies incorporating drugs other than PARP inhibitors are needed to treat incurable prostate cancer. As such, a strategic development of drugs targeting PI3K/AKT/mTOR pathway is necessary. The mammalian target of rapamycin (mTOR) is expressed in the representative prostate cancer cell lines such as DU145, PC3, LNCaP, and 22RV1 (Figure 1). According to a review article published in 2016, Park and his colleagues found that mTOR inhibitors have limited effect on prostate cancer. However, studies published afterwards have provided a different point of view.
\end{abstract}

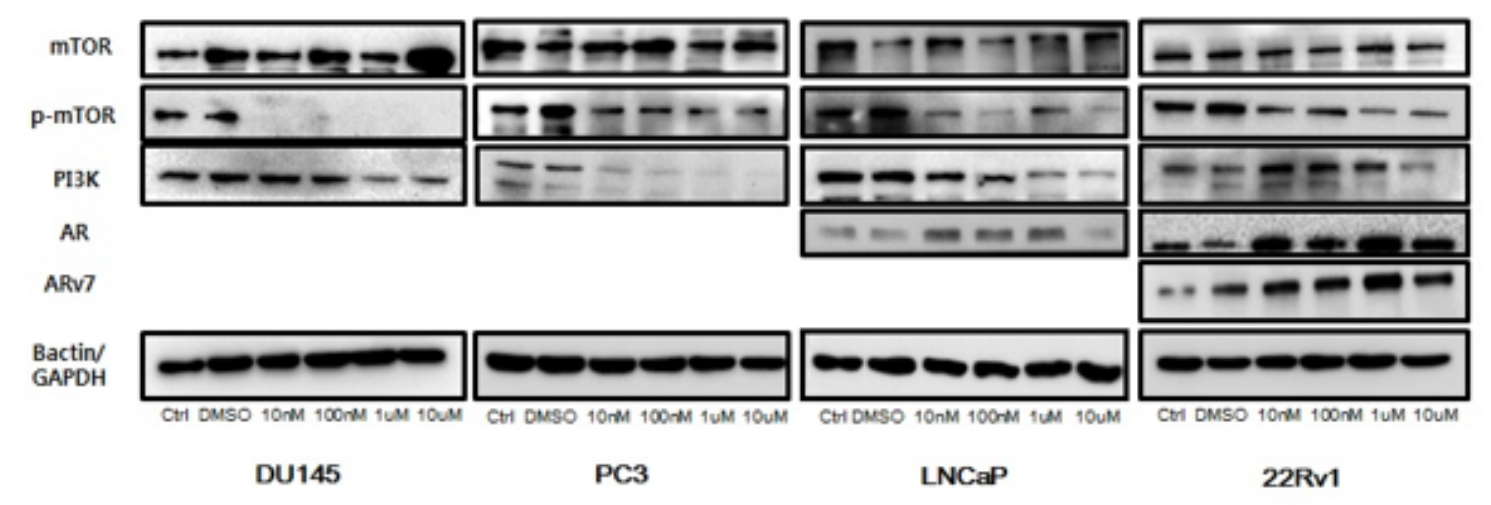

Figure 1: Western blot analysis of dose-dependent mTOR signaling pathways in prostate cancer cell lines. 


\section{Introduction}

Liss et al.[1] reported that mTOR inhibitors can slow disease progression in low-risk prostate cancer patients [1]. This finding proposed lengthening or sustaining of the surveillance period by slowing disease progression in patients under active surveillance. Hsieh et al.[2] have shown that mTOR can mediate the translation of pro-invasion mRNAs in prostate cancer cells, indicating that mTOR plays an important role in prostate cancer invasion and metastasis [2]. This study demonstrated that mTOR inhibitors may be beneficial in preventing prostate cancer metastasis. Han et al. reported the development of prostate cancer though AKT/mTOR inhibition and proposed a possible treatment strategy through AKT/mTOR pathway activation [3].

Russo et al. described the relationship between mTOR expression and intra-tumoral heterogeneity, one of the major characteristics of prostate cancer. This study presented mTOR parameter as a biomarker for intra-tumoral heterogeneity [4]. Walsh et al. described a regulatory complex of the androgen receptor and mTOR, describing its role in promoting SREBF1 expression [5].

Bono et al. reported excellent results using a combination therapy of abiraterone acetate and AKT inhibitor targeting the PI3K/ AKT/mTOR pathway in metastatic CRPC patients. A randomized phase II study has evaluated the efficacy of adding ipatasertib, an AKT inhibitor in patients with and without PTEN loss [6].

L. Braglia et al. [7] also described the role of AKT inhibitors in the PI3K/AKT/mTOR pathway in CRPC patients with and without PTEN loss [7]. Lin et al. [8] reported the upregulation of FOXM1 expression in mCPRC patients and revealed that FOXM1 targets AMPK/mTOR to activate the autophagy pathway, which affects the response to docetaxel, providing the basis for a new treatment strategy [8].

In recently published research, drugs or biomarkers targeting PI3K/AKT/mTOR pathway in CRPC patients may be used to establish a new treatment strategy for CRPC patients. The discovery and development of new drugs should be followed through further studies and drug development research.

\section{Acknowledgement}

Grant/Fund Support: This article was funded by National Research Foundation (NRF2019R1G1A1).

\section{References}

1. Michael A Liss, Lanette Rickborn, John DiGiovanni, Dean Bacich, Linda A (2018) DeGraffenried, Manish Parihar, Ian M. Thompson, Zelton Dave Sharp. mTOR Inhibitors for Treatment of Low-Risk Prostate Cancer. Med Hypotheses 117: 63-68.

2. Andrew C Hsieh, Yi Liu, Merritt P Edlind, Nicholas T Ingolia, Matthew $\mathrm{R}$ Janes, et al. (2012) The translational landscape of mTOR signalling steers cancer initiation and metastasis. Nature 485(7396): 55-61.

3. Yu Han, Chao Liu, Dongfang Zhang, Hongchao Men, Lifang Huo, et al. (2019) Mechanosensitive ion channel Piezo1 promotes prostate cancer development through the activation of the Akt/mTOR pathway and acceleration of cell cycle. Int J Oncol 55(3):629-644.

4. Giorgio Ivan Russo, Jörg Hennenlotter, Ulrich Vogel, Ursula Kühs, Thomas Manfred Wurm, et al. (2019) Intratumoral Heterogeneity Determines the Expression of mTOR-pathway Proteins in Prostate Cancer. Dis Markers 2019: 1296865.

5. Étienne Audet-Walsh, Mathieu Vernier, Tracey Yee, Chloé Laflamme, Susan Li, et al. (2018) Activity Is Regulated by an AR/mTOR Nuclear Axis in Prostate Cancer. Mol Cancer Res 16(9): 1396-1405.

6. Johann S de Bono, Ugo De Giorgi, Daniel Nava Rodrigues, Christophe Massard, Sergio Bracarda, et al. (2019) Randomized Phase II Study Evaluating Akt Blockade with Ipatasertib, in Combination with Abiraterone, in Patients with Metastatic Prostate Cancer with and without PTEN Loss. Clin Cancer Res 25(3): 928-936.

7. Luca Braglia, Manuela Zavatti, Marco Vinceti, Alberto M Martelli, Sandra Marmiroli (2020) Deregulated PTEN/PI3K/AKT/mTOR signaling in prostate cancer: Still a potential druggable target? Biochim Biophys Acta Mol Cell Res. Sep;1867(9):118731.

8. Jian-Zhong Lin, Wei-Wan Wang, Ting-Ting Hu, Gang-Yi Zhu, Li-Nan Li, et al. (2020) FOXM1 contributes to docetaxel resistance in castrationresistant prostate cancer by inducing AMPK/mTOR-mediated autophagy. Cancer Lett 469: 481-489. 